\title{
Uso de tecnología para acercar al ciudadano a su local de votación
}

\author{
Mariano Cucho Espinoza* \\ Oficina Nacional de Procesos Electorales (ONPE). Lima, Perú \\ Recibido: 16 de marzo del 2017 / Aprobado: 26 de abril del 2017
}

Resumen: El presente artículo ha mostrado el origen, desarrollo y logros de la aplicación Elige tu Local de Votación (ETLV), una innovación que llevó a cabo la Oficina Nacional de Procesos Electorales (ONPE) entre los años 2015-2016 como parte de su enfoque de modernización tecnológica. Se hizo un recuento de cómo se gestionó internamente este software, las gerencias comprendidas y la planificación que requirió. Además, se enfatizó la forma en que se organizó el recurso humano y el papel de la alta dirección. El artículo ha culminado con un recuento de los beneficios que esta innovación ha generado en la ciudadanía.

Palabras clave: innovaciones tecnológicas / tecnología electoral / elecciones / inclusión electoral / voto electrónico / ONPE

\section{Use of technology to bring citizens to their polling place}

ABstRaCT: This article has shown the origin, development and achievements of the applicative Elige tu Local de Votación (Choose your Voting Location), an innovation developed by the National Office of Electoral Processes (ONPE, for its acronym in Spanish) between 2015-2016, as part of its technological modernization approach. It includes a review of how this software was managed internally, the offices at charge and the planning required. In addition, the way in which human resources were organized and the role of senior executive was emphasized. The article ends with a report of the benefits to citizens produced by this innovation.

Keywords: technological innovations / electoral technology / elections / electoral inclusion / electronic voting / ONPE

* Correo electrónico: Mcucho2006@gmail.com 


\section{INTRODUCCIÓN}

Con el avance imparable de las tecnologías de la información y comunicaciones, así como con la creciente demanda de un Estado moderno por parte de la ciudadanía, surge el reto y al mismo tiempo la oportunidad para los organismos estatales de emprender el camino del cambio. La ONPE no se ha quedado atrás en este esfuerzo y ha logrado desarrollar varias innovaciones tecnológicas con el fin de contribuir al fortalecimiento de la democracia en el país.

\section{LA INNOVACIÓN TECNOLÓGICA EN EL MARCO DE LA MODERNIZACIÓN DE LA GESTIÓN PÚBLICA EN LA ONPE}

En los últimos años, la ONPE ha tenido el reto de continuar aportando al desarrollo del sistema democrático peruano mediante el cumplimiento de su misión institucional -la ejecución de procesos electorales y de consulta- y a su vez con la implementación de un conjunto de mejoras orientadas a la modernización de su gestión interna. Esto último obedece a los lineamientos de la Política Nacional de Modernización de la Gestión Pública (PNMGP) a los que se adhiere (Presidencia del Consejo de Ministros [PCM], 2013). En ellos se enfatiza la necesidad de usar la tecnología para mejorar los procesos internos y los servicios ofrecidos a la ciudadanía.

De acuerdo con ese esquema, la organización ha ampliado en los últimos años su cartera de servicios y le ha incorporado mejoras, entre ellas y cada vez más una serie de innovaciones y soluciones tecnológicas. Esto no sería posible sin la planificación estratégica, que ha permitido definir cinco ejes claves para la modernización institucional de la ONPE, los que se basan en la incorporación de soluciones tecnológicas:

1. Nuevo modelo de gestión. Incorporación de un sistema de planificación y gestión operativa por procesos y resultados, así como un sistema de seguridad de la información.

2. Tecnología de punta. Desarrollo de una plataforma tecnológica moderna para los diferentes servicios ofrecidos por la organización.

3. Voto electrónico. Gestión de la implementación progresiva del voto electrónico presencial y no presencial.

4. Gestión humana. Uso de los nuevos soportes tecnológicos para la capacitación y la mejora continua del personal.

5. Infraestructura. Dotación de adecuado equipamiento tecnológico a los espacios de la institución, en función de los servicios ofrecidos. 
A la fecha, el trabajo de la ONPE, según los lineamientos descritos, ha producido logros notables. No solamente se trata del voto electrónico -una innovación que en las elecciones generales 2016 se expandió a un total de 19 distritos (ONPE 2016a) -, sino también del sistema de escrutinio automatizado (SEA). Este se usa en la crítica etapa final de la jornada electoral y elimina, gracias a la tecnología, los errores manuales en las actas y, por tanto, las actas observadas. También cabe mencionar la capacitación virtual de los actores electorales, una aplicación que, a través de la tecnología y las redes, permite llegar a una mayor cantidad de personas; y la aplicación Verifondos-Aportes Limpios, que facilita al público interesado la revisión de los aportes de campaña. A esto se debe sumar todo lo que la ciudadanía no ve directamente, pero que resulta crucial para la gestión interna de la ONPE y en consecuencia para la eficiencia de sus servicios. Así, la tecnología se ha empleado para la conversión de documentación física en microformas digitales, las cuales han sido archivadas digitalmente para ahorrar espacio y papel. La economía de este insumo también es uno de los beneficios del sistema de gestión documental (SGD), que hace posible el envío y recepción de documentos por la interface digital.

En general, las innovaciones tecnológicas han repercutido en todo el ámbito de la organización y han traído cambios importantes que merecen ser de conocimiento de la ciudadanía. Sin embargo, no debe olvidarse que toda mejora tecnológica implementada en el ámbito electoral, por más pequeña o ambiciosa que sea, debe orientarse hacia el fin máximo de una organización como la ONPE: la ejecución de los procesos electorales y por consiguiente la contribución al mantenimiento y fortalecimiento del sistema democrático. En los últimos años, este es el espíritu que ha guiado para la incorporación de tecnología en las labores de la institución, la noción de que modernizar la gestión y servicios de la ONPE ayuda a modernizar nuestra democracia.

Una de las innovaciones que se llevaron a cabo en las elecciones generales 2016 fue la aplicación ETLV. Esta, por primera vez, hizo posible que algunos electores del país eligieran el lugar donde podían ejercer su voto, por lo que sentó un cambio de trascendental importancia para futuros procesos electorales. La forma en que se diseñó e implementó la aplicación es una muestra del continuo esfuerzo de la ONPE por avanzar hacia una modernización institucional, reflejada en mejores y más accesibles servicios para la ciudadanía durante los procesos electorales. 


\section{LA APLICACIÓN “ELIGE TU LOCAL DE VOTACIÓN”: UN LOGRO DE LA INNOVACIÓN EN LA ONPE}

Elige tu Local de Votación (ETLV) es una aplicación web que hace posible que los ciudadanos elijan el local donde desean ejercer su voto en un proceso electoral. El aplicativo se elaboró considerando el problema del tiempo invertido por los electores durante el desarrollo de las jornadas electorales para trasladarse a locales de votación lejanos de su residencia, junto con otras dificultades relacionadas, tales como la congestión vehicular - y la mayor contaminación ambiental que esta genera-, la separación de las familias al tener que votar en locales distintos y las tardanzas de los ciudadanos, producto de los factores mencionados.

Tomando en cuenta lo anterior, la aplicación ETLV - que es accesible a través de internet ya sea por una PC o por teléfono celular- hizo posible que el elector seleccionara hasta tres alternativas de locales de su preferencia. Sus opciones fueron procesadas y luego se le informó del local definitivo vía correo electrónico o por la página web. Este es otro ejemplo de cómo la tecnología facilita el ejercicio de un derecho ciudadano fundamental como es el voto, acerca a las familias durante el día de la votación y convierte efectivamente la jornada en una fiesta democrática.

Para hacer realidad ETLV, se siguieron tres fases bien delimitadas, desarrolladas entre los años 2015 y 2016:

- Desarrollo e implementación de la aplicación web

- Capacitación de los recursos humanos

- Trabajo de campo

\subsection{Desarrollo e implementación de la aplicación web}

Para conseguir que ETLV fuera una realidad, se llevó a cabo un intenso trabajo de planificación, el cual abarcó la toma de decisiones entre la alta dirección, las gerencias de ONPE que participaban de la iniciativa y colaboradores concretos, quienes intervinieron en la programación de la aplicación, el diseño de su interface y otras de sus características técnicas.

El punto de partida del proyecto provino de la constatación de una necesidad en los electores. Específicamente, las dificultades que suelen experimentar el día de la jornada electoral, especialmente por las grandes distancias que deben recorrer entre su domicilio y el local de votación. Esta situación genera congestionamiento vehicular, pérdida de horas 
hombre, gastos en transporte, seguridad, entre otros, y se convierte en un problema generalizado que afecta particularmente a los electores que residen en las zonas periféricas de Lima metropolitana y el Callao.

La idea de la aplicación nació al contrastar las problemáticas señaladas, junto con las soluciones que se habían implementado para la instalación de mesas en los centros poblados, una labor que le corresponde a la Gerencia de Planeamiento y Presupuesto (GPP) de la ONPE. De acuerdo con la experiencia de otros procesos electorales, como las elecciones regionales y municipales 2014 (ERM 2014), se sabe que muchos electores recorren grandes distancias para llegar al local de votación asignado. Esta dificultad se acentúa en el interior del país debido a lo extenso del territorio, la diversidad geográfica en las tres regiones (costa, sierra y selva), el limitado transporte público, etc. Como consecuencia, los electores experimentan grandes dificultades para desplazarse hacia su local de votación cuando este se encuentra en lugares de difícil acceso; peor aun, muchos deciden no ir a votar, lo que les ocasiona no solo la imposición de una multa, sino también la vulneración del derecho a elegir a sus autoridades. Esto afecta la participación del ciudadano en el proceso electoral.

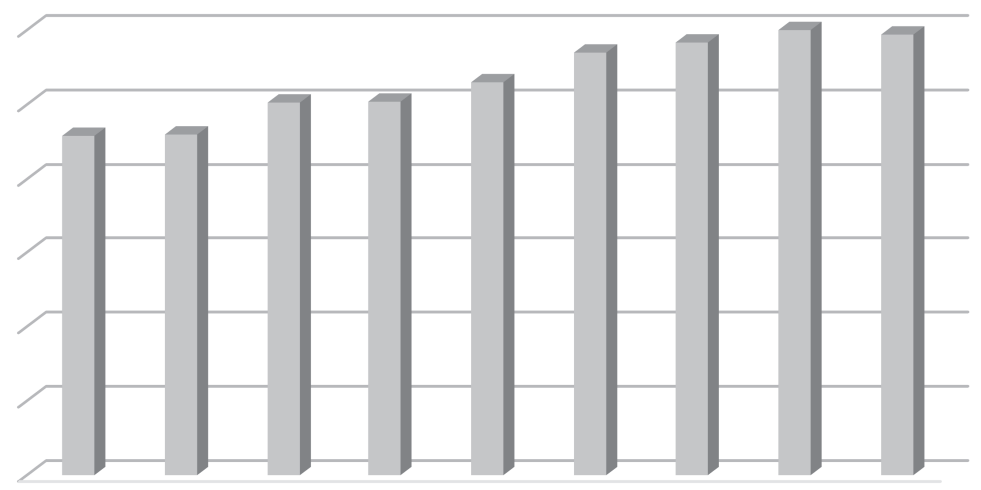

Figura 1. Porcentaje de la población de seis años a más que usaba internet en Lima Metropolitana

Fuente: Encuesta Nacional de Hogares (ENAHO), INEI (2015) 
De otro lado, se contó con información estadística actualizada que daba cuenta de la creciente incorporación de las TIC en la población peruana, lo cual significó una oportunidad para aprovechar el potencial de la creciente familiaridad de la gente con las nuevas tecnologías. En efecto, según el INEI (2015), el 62,4 \% de la población de seis años a más, de Lima Metropolitana, usaba habitualmente internet. Asimismo, cerca del $50 \%$ de los limeños cuenta hoy con acceso a internet desde sus casas, de modo que es más cotidiano el acceso a las TIC.

Tomando en cuenta estos datos, se concibió implementar la aplicación ETLV en algunas zonas de Lima Metropolitana y Callao. Para esto, se priorizaron bolsones de población y zonas a partir de los siguientes criterios, definidos por la Gerencia de Planeamiento y Presupuesto en su planificación:

- La población electoral. Distritos que concentran una mayor población de electores.

- La concentración de pueblos jóvenes. Asentamientos humanos ubicados en la periferia y con dificultades de acceso.

- La escasez de medios de transporte. La poca disponibilidad de medios de transporte durante la jornada electoral y el encarecimiento de sus precios, un problema que afecta principalmente a las zonas periféricas.

- La situación económica. La concentración de población de escasos recursos en las zonas señaladas.

- La inseguridad ciudadana. El riesgo que implica para los electores trasladarse hacia un local de votación alejado de su domicilio.

- La infraestructura. Aun con todas las limitaciones de los distritos intervenidos, hay al menos un centro educativo que puede ser utilizado como local de votación por el electorado del lugar.

En términos de la planificación, ETLV es una iniciativa que se alineó desde el inicio con el objetivo estratégico 1, "Incrementar gradual y progresivamente el voto electrónico y la modernización del proceso electoral", según el cual la Alta Dirección impulsa todas las iniciativas de mejora e innovación de procesos y proyectos. Así, la Alta Dirección de la ONPE encargó a la Gerencia de Planeamiento y Presupuesto (GPP) la construcción de una iniciativa para mejorar la distribución de los locales de votación. Esta coordinó con la Gerencia de Informática y Tecnología Electoral (GITE) el desarrollo de una aplicación web que permitiera a los electores elegir su local de votación. La idea era usar la información 
proporcionada por los electores para construir un nuevo sistema de asignación de los locales de votación, según sus preferencias ciudadanas. El proyecto de mejora se tituló originalmente ETLV. Tu Voto Más Cerca de Casa, y tuvo como miras lograr cinco beneficios específicos:

- Acercamiento del elector al local de votación

- Ahorro de tiempo y costo

- Seguridad en el traslado al local de votación

- Evitar la generación del tráfico vehicular

- Promover el voto familiar

Debido a la envergadura del proyecto, fue necesario establecer una metodología para ajustar adecuadamente su diseño, de forma que respondiera de la manera más eficiente a las necesidades que le dieron origen. Por ello, la maduración del proyecto requirió la elaboración de la metodología análisis del problema e identificación de alternativas de solución, la cual requirió los siguientes instrumentos de planificación:

- Análisis FODA: identificación de la situación actual

- Árbol de problemas: identificación del problema central y el efecto final

- Árbol de objetivos: identificación del objetivo principal y el fin último de la solución propuesta

- Matriz de marco lógico de proyecto: definición de alternativas de solución y estrategias

En la construcción del FODA un insumo clave para quienes llevaron a cabo la planificación fue la experiencia de la ONPE en la atención realizada de forma permanente a los centros poblados. Se sabe que los electores de los centros poblados son atendidos en su localidad y es necesario el despliegue de recursos humanos y de logística para empadronar al elector in situ, mediante una serie de procesos, autorizaciones y verificaciones. De esta forma, el electorado ya no tiene que trasladarse hasta el distrito capital del centro poblado durante la jornada electoral y así se limita un factor que incide en el ausentismo durante la elección. Con esta misma concepción de atención, proveniente del espacio rural, se pensó que el electorado del ámbito urbano también podría experimentar un acercamiento del local de votación a sus domicilios. La viabilidad del ETLV, por tanto, fue evaluada de acuerdo con la posibilidad o no de efectuar tal acercamiento. Así, el problema principal identificado en el árbol de problemas y respecto del cual se dirigieron las soluciones del 
árbol de objetivos fue el alejamiento del local de votación del domicilio de los electores. Esta situación se enfocó en el caso de los electores del distrito de Mi Perú en el Callao, circunscripción que, por tanto, sería la pionera en el desarrollo del proyecto.

Cuando la propuesta estuvo lista, fue presentada en reunión de gerencia a la Alta Dirección. La aplicación ETLV se dividía en dos módulos: a) módulo ciudadano, a través del cual los electores acceden desde cualquier computadora con internet y eligen el local de votación más cercano a su domicilio; b) módulo gestor, por medio del cual los electores tienen un contacto directo con el personal de la ONPE (equipos itinerantes), quienes registran a los electores en la aplicación ETLV, que fue aprobada en Comité de Gerencia, tal como consta en el Acta $N^{\circ}$ 000021-2015CG del 1 de setiembre del 2015 (ONPE 2015). De esta manera, primero se decidió implementar ETLV en el recientemente creado distrito de Mi Perú, con miras a las elecciones municipales que se desarrollarían en esta comuna en noviembre del 2015. A partir de los resultados de $\mathrm{Mi}$ Perú, se contempló posteriormente extender la aplicación a toda Lima Metropolitana para las elecciones generales 2016.

Si bien el origen de la iniciativa comprendió dos gerencias (GPP y GITE) y la Alta Dirección (la Jefatura Nacional), las demás gerencias de la ONPE también apoyaron de diferentes maneras su puesta en marcha.

El equipo del proyecto estuvo integrado por especialistas (informáticos y diseñadores), además de funcionarios de la Gerencia de Planeamiento y Presupuesto (GPP). La comunicación horizontal y transversal dentro de la ONPE facilitó que el equipo obtuviera los recursos internos necesarios. Para ello, fue clave el tejido comunicacional, establecido gracias al sistema de gestión documental (SGD), los correos electrónicos, así como las comunicaciones escritas y directas. Estas últimas se dieron en las reuniones de despacho entre gerentes y la Jefatura Nacional.

La comunicación del equipo del proyecto con la GPP se garantizó mediante reuniones periódicas para la revisión de los avances de la iniciativa, según un cronograma de trabajo. En tales reuniones se evaluaron aspectos técnicos y de diseño de la aplicación, de forma que el producto tuviese un adecuado consenso entre todas las partes involucradas. Asimismo, la Alta Dirección fue informada permanentemente sobre el proyecto y se recogieron recomendaciones y sugerencias que sirvieron para la retroalimentación constante de la iniciativa. 
Tabla 1

Gerencias comprendidas en el desarrollo de la aplicación ETLV

\begin{tabular}{ll}
\hline \multicolumn{1}{c}{ Gerencias } & \multicolumn{1}{c}{ Apoyo } \\
\hline $\begin{array}{l}\text { La Gerencia de } \\
\text { Organización Electoral y } \\
\text { Coordinación Regional } \\
\text { (Goecor) }\end{array}$ & $\begin{array}{l}\text { Contrató 17 asistentes para ejecutar las campañas de } \\
\text { difusión y registro de electores en los puntos ubicados en } \\
\text { los lugares públicos de Lima Metropolitana y Callao. }\end{array}$ \\
\hline $\begin{array}{l}\text { La Gerencia General } \\
\text { (GG) }\end{array}$ & $\begin{array}{l}\text { Realizó las coordinaciones necesarias con entidades } \\
\text { públicas y privadas a través de la entrega de oficios, en los } \\
\text { cuales solicitó el apoyo en la difusión de la aplicación ETLV. }\end{array}$ \\
\hline $\begin{array}{l}\text { La Gerencia de } \\
\text { Comunicaciones y } \\
\text { Relaciones Corporativas } \\
\text { (GCRC) }\end{array}$ & $\begin{array}{l}\text { Llevó a cabo el plan de medios con fines de difusión y } \\
\text { motivación para la campaña Elige tu Local de Votación }\end{array}$ \\
\hline $\begin{array}{l}\text { La Oficina de Seguridad } \\
\text { y Defensa Nacional } \\
\text { (OSDN) }\end{array}$ & $\begin{array}{l}\text { Solicitó seguridad policial al director general de la Policía } \\
\text { Nacional del Perú para garantizar la seguridad del personal } \\
\text { y de los equipos informáticos hasta culminar las campañas } \\
\text { de difusión y registro de los electores del programa ETLV. }\end{array}$ \\
\hline $\begin{array}{l}\text { La Gerencia de } \\
\text { Administración (GAD) }\end{array}$ & $\begin{array}{l}\text { Gestionó la asignación de recursos para brindar refrigerio y } \\
\text { movilidad al personal que prestó apoyo en las campañas de } \\
\text { difusión y registro de electores del programa ETLV. }\end{array}$ \\
\hline $\begin{array}{l}\text { La Gerencia de } \\
\text { Electoral (GIEE) }\end{array}$ & $\begin{array}{l}\text { Elaboró el manual del usuario Elige tu local de votación } \\
\text { (aplicación piloto en Mi Perú), el cual ayudó a los } \\
\text { ciudadanos (usuarios) para que pudieran acceder al } \\
\text { programa virtual con el fin de registrar su local de votación. }\end{array}$ \\
\hline
\end{tabular}

Fuente: ONPE (2016b)

\subsection{Capacitación de los recursos humanos}

Una vez validada la innovación virtual, el siguiente paso fue llevar a cabo su difusión. Para ello, fue necesario acercar la aplicación a la ciudadanía con el fin de que se hiciera conocida y comenzara a ser usada por los propios electores.

Surgieron dos requerimientos de capacitación para la implementación del proyecto. El primero, el conocimiento de las herramientas de calidad para la mejora de los procesos y proyectos. Con este fin, se programó el taller "Herramientas de calidad" para los gestores y 
personal de apoyo directo, a fin de que el proyecto pudiera ser conceptualizado y planificado de forma adecuada.

La segunda necesidad de capacitación fue requerida para el personal que realizó trabajo de campo, a quienes se les indicó cómo debían llevar a cabo el registro de electores in situ con el módulo gestor de la aplicación ETLV.

De crucial importancia para el éxito de las capacitaciones fue determinar, mediante evaluaciones, la brecha existente en los conocimientos, experiencias y habilidades entre cada miembro del equipo. De esta forma, fue posible identificar los aspectos que deberían ser mejorados del perfil de los integrantes. En general, la secuencia seguida para las evaluaciones fue la siguiente: a) recopilación de perfiles de cada uno de los miembros, b) revisión y clasificación de los perfiles, y c) evaluaciones del personal.

\subsection{Trabajo de campo}

Finalmente, fue necesario trasladar al personal capacitado para desarrollar actividades de campo con el fin de difundir el programa virtual y usarlo in situ con la ciudadanía. El trabajo de campo se planificó cuidadosamente y consistió en varias jornadas de trabajo entre el 13 y el 27 de setiembre en el distrito de Mi Perú; y posteriormente entre diciembre y enero del 2016 con miras a las elecciones generales del 2016.

El primer día del trabajo de campo en el distrito de Mi Perú, un grupo de colaboradores de la ONPE conformado por diez personas -incluyendo miembros de la Alta Dirección-partió hacia el nuevo distrito. Ahí comenzaron con las labores de difusión repartiendo volantes a los transeúntes, donde se informaba al elector cómo podía elegir su local de votación. En paralelo, otros integrantes del equipo instalaron 10 laptops con acceso a internet, para iniciar el registro de los primeros electores.

Fue así como en el distrito de Mi Perú los electores pudieron, por primera vez, escoger el local de votación de su preferencia. Al finalizar la primera jornada de difusión (el 27 de setiembre), se consiguió que el $71,5 \%$ de la población electoral empadronada del distrito -aproximadamente 14192 de 18922 electores-designaran su local de votación mediante dicha aplicación (ONPE, 2015). 


\section{2}

Orientador

Informar al ciudadano sobre los locales de votación disponibles previamente al registro, en el punto de registro.

\section{1}

Promotor

Difundir, promocionar e informar sobre el registro en el área de intervención.

\section{Registrador}

Acceder al aplicativo e ingresar la información del ciudadano en el aplicativo ETLV, en el punto de registro.

Figura 2. Proceso de atención al usuario en la ejecución presencial de la aplicación ETLV Fuente: ONPE (2016b)

En el caso del trabajo de campo para las elecciones generales 2016, desarrollado entre diciembre del 2015 y enero del 2016, se desplegó una mayor cantidad de equipos de capacitación, dado que había más distritos en los cuales generar incidencia. Asimismo, fue necesario coordinar con 30 municipios distritales de Lima y Callao, así como con establecimientos privados (centros comerciales, por ejemplo) donde se contempló llevar a cabo las acciones de capacitación. Ello permitió contar con apoyo logístico e infraestructura para la puesta en marcha de la estrategia de registro in situ de los electores en diferentes establecimientos (Tabla 2).

El trabajo de campo para el registro de los electores de Lima Metropolitana en estos diferentes espacios culminó el 18 de enero del 2016. Después de las arduas labores de difusión e inscripción presencial efectuadas por los colaboradores de la ONPE, se consiguió registrar 2224523 electores (29,3\% del total de la población electoral de Lima Metropolitana y Callao); se había superado la meta propuesta de 1903816 electores. Por ello, la implementación del programa virtual ETLV en el campo ya se vislumbraba como un éxito en el momento en que se cerró el registro previo a las elecciones del 2016. Las percepciones de la ciudadanía que usó la aplicación tanto en Mi Perú como en los diferentes sectores donde se realizó el registro presencial de electores para las elecciones generales 2016 coincidían en mostrar una valoración positiva y satisfacción respecto del servicio ofrecido. 
Tabla 2

Organizaciones con las que se coordinó para trabajo de campo de ETLV en Lima, por distrito

\begin{tabular}{|c|c|}
\hline Ate & $\begin{array}{l}\text { Agencia Municipal de Huaycán } \\
\text { Agencia Municipal de Santa Clara } \\
\text { Centro Comercial Real Plaza de Santa Clara }\end{array}$ \\
\hline Breña & Hospital del Niño \\
\hline Santiago de Surco & Centro Comercial Jockey Plaza \\
\hline Cercado de Lima & $\begin{array}{l}\text { Universidad Nacional Mayor de San Marcos } \\
\text { Centro Comercial Real Plaza del Centro Cívico } \\
\text { Hospital Arzobispo Loayza } \\
\text { Plaza San Martín }\end{array}$ \\
\hline Jesús María & Centro Comercial Real Plaza de Salaverry \\
\hline Lince & Hospital Edgardo Rebagliati Martins \\
\hline San Miguel & Centro Comercial Plaza San Miguel \\
\hline Chorrillos & Centro Comercial Plaza Lima Sur \\
\hline Independencia & $\begin{array}{l}\text { Centro Comercial Megaplaza } \\
\text { Centro Comercial Plaza Norte }\end{array}$ \\
\hline San Martín de Porres & $\begin{array}{l}\text { Centro Comercial Pro } \\
\text { Mercado Unicachi Pro }\end{array}$ \\
\hline Pueblo Libre & Hospital Santa Rosa \\
\hline Bellavista & $\begin{array}{l}\text { Centro Comercial Mall Aventura Plaza } \\
\text { Hospital Daniel Alcides Carrión }\end{array}$ \\
\hline La Molina & Tienda San Jorge \\
\hline La Victoria & $\begin{array}{l}\text { Parque Cánepa } \\
\text { Hospital Guillermo Almenara } \\
\text { Emporio Comercial de Gamarra }\end{array}$ \\
\hline San Juan de Miraflores & Mercado Ciudad de Dios \\
\hline Callao Cercado & Centro Comercial Minka \\
\hline
\end{tabular}

Fuente: ONPE (2016b) 


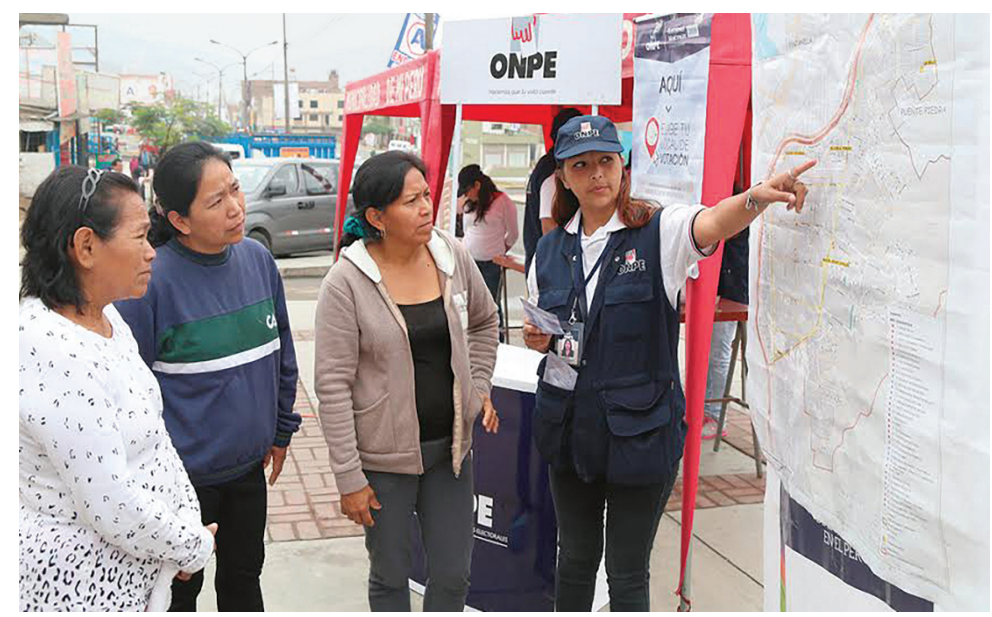

Figura 3. Trabajo de campo para el registro de la ciudadanía a través de la aplicación Elige tu Local de Votación

Fuente: ONPE (2016c)

\section{LOS LOGROS DE “ELIGE TU LUGAR DE VOTACIÓN” Y EL FUTURO DE LA APLICACIÓN}

En suma, el programa virtual ETLV apunta a los principales clientes externos de la ONPE, los electores, quienes gracias a la tecnología ahora pueden votar más cerca de su residencia, más libres del congestionamiento vehicular y más cerca de sus familias; además, disponen de una mayor libertad y capacidad de agencia en tanto ahora pueden decidir dónde ejercer su derecho democrático al voto. Esta es solo una muestra de cómo la tecnología, aplicada a un organismo electoral, como la ONPE, puede ser una aliada importante en la mejora de la democracia. Hoy, se puede decir que las innovaciones tecnológicas son fundamentales para que la ONPE continúe haciendo que el voto de los peruanos y las peruanas cuente.

En este artículo se ha hecho un repaso del origen, desarrollo y resultados preliminares de la aplicación; además, se ha destacado su alineación con el enfoque de modernización institucional de la ONPE. Es necesario precisar que, desde el punto de vista de gestión de la innovación, un elemento crucial para el logro del proyecto fue contar con las facilidades otorgadas por la Alta Dirección para promover el trabajo del equipo en la ejecución del proyecto. Entre estas disposiciones se encuentran las siguientes: 
- Dio asignación presupuestal para la adquisición de los medios operativos y los recursos humanos para el proyecto.

- Aprobó y monitoreó la puesta en marcha de la aplicación. La Alta Dirección designó personal técnico de GITE para brindar soporte tecnológico para el alojamiento y publicación de la plataforma virtual en los servidores web, así como asesoría en la definición e implementación del programa virtual.

- Garantizó la logística e infraestructura para los integrantes del equipo de trabajo (mobiliario, equipos y materiales de trabajo).

- Dio disponibilidad de horario a los integrantes del equipo de trabajo en las actividades de evaluación, coordinación y monitoreo del servicio de virtualización.

- Apoyó en las tareas operativas de campo durante la recolección de los datos. En dichas labores pudo comprobar las dificultades que se estaban afrontando, por lo que junto con el equipo tomaron las decisiones correctivas para que el proyecto fuera un éxito.

- Solicitó a la Gerencia de Comunicaciones y Relaciones Corporativas (GCRC) la difusión del proyecto a través de diversos medios, como redes sociales, perifoneo en el distrito de Mi Perú y entrega de material de difusión a los electores.

- Se preocupó por que el equipo del proyecto contara con los medios operativos logísticos y de alimentación necesarios para las jornadas de campo.

Viendo en perspectiva la implementación de la aplicación ETLV tanto para las elecciones en Mi Perú como para las elecciones generales 2016, los logros de esta innovación tecnológica electoral se pueden resumir en función de los cinco beneficios que originalmente se proyectaron:

- Acercamiento del elector al local de votación, según su lugar de residencia. La aplicación permite que el elector sufrague en el local de votación más cercano a su domicilio, en el distrito de su residencia registrado en el DNI. Como consecuencia de este acercamiento, tiene incluso la posibilidad de ir caminando a su local de votación. Esto se reflejó en las consultas realizadas por el personal de ONPE a los electores de Mi Perú durante las elecciones del 29 de noviembre de ese distrito. Se trató de un logro notable, dado que los electores fueron caminando y ya no dependieron del transporte público, con lo cual el tráfico se habría reducido. 
- Ahorro de tiempo y costo. Como ya se ha dicho, el acercamiento del local de votación al domicilio del elector genera que este ahorre tiempo al desplazarse tanto de ida como de vuelta.

- Seguridad en el traslado al local de votación. El elector elige el local cerca de su domicilio, que es un lugar familiar, en vez de desplazarse a zonas desconocidas.

- Evitar la generación del tráfico vehicular. Al estar cerca de su local y desplazarse con la familia, el elector ve viable la opción de dirigirse a pie y así ayuda a la disminución del tráfico vehicular.

- Promover el voto familiar. Varios miembros de una familia pueden elegir votar en el mismo local de votación, de forma que van y regresan juntos. Esto genera unión en el hogar antes, durante y después de ejercer el voto.

- El elector conoce antes del día de la jornada electoral el lugar donde sufragará. El ciudadano al momento de elegir su local de votación, se informa de su ubicación y se percata de que la zona es segura, dado que por lo general es un espacio conocido. Ello conlleva a que conozca de antemano la ruta más eficaz y segura para desplazarse hasta su local, y evita extraviarse, sufrir asaltos u otro tipo de percance que puedan influir en el ausentismo el día de la jornada electoral.

Así, se entiende que tras los resultados positivos obtenidos en $\mathrm{Mi}$ Perú y en Lima Metropolitana y Callao, la aspiración de la ONPE ahora es ampliar la cobertura de ETLV a una mayor cantidad de distritos y electores para las elecciones regionales y municipales 2018. De acuerdo con lo planificado por la Alta Dirección, se abriga la expectativa de que al 2018 la aplicación pueda extenderse a los 1875 distritos del país y abarque a los 23 millones de electores que tentativamente compondrían el padrón electoral de los futuros comicios.

De esta forma, en un futuro cercano la mayor parte de los electores del Perú incorporarán plenamente un nuevo derecho a su vida democrática: el de poder elegir dónde votar. Así, ampliarán el conjunto de los derechos democráticos de los que hoy goza la población peruana. Por ello, ETLV no es solamente una solución tecnológica más, sino que constituye una muestra de cómo la tecnología moderna, aplicada al campo electoral, puede realmente enriquecer el sistema y los valores democráticos. 


\section{REFERENCIAS}

Instituto Nacional de Estadística e Informática. (2015). Base de datos de la Encuesta Nacional de Hogares (ENAHO). Recuperado de https://www.inei.gob.pe/bases-de-datos

Oficina Nacional de Procesos Electorales. (2014a). Plan Estratégico Institucional 2014-2017. Recuperado de https://www.web.onpe. gob.pe/modMarco-Legal/Resoluciones/RJ-064-2014.pdf

Oficina Nacional de Procesos Electorales. (2014b). Voto electrónico y desarrollo de las tecnologías de la información y la comunicación en el Perú. Lima: Autor.

Oficina Nacional de Procesos Electorales. (2014c). El voto electrónico en la práctica: perspectivas y dinámicas desde la experiencia de las elecciones regionales y municipales 2014. Lima: Autor.

Oficina Nacional de Procesos Electorales. (2016a). Plan Operativo para las Elecciones General 2016. Recuperado de https://www.web. onpe.gob.pe/modMarco-Legal/Resoluciones/RJ-392-2015.pdf

Oficina Nacional de Procesos Electorales. (2016b). Informe del proyecto Elige tu Local de Votación. Lima: Gerencia de Planeamiento y Presupuesto de la ONPE.

Oficina Nacional de Procesos Electorales. (2016c). Innovaciones tecnológicas para la democracia. Lima: Autor.

Oficina Nacional de Procesos Electorales. (2016d). Memoria institucional 2013-2016. Lima: Autor.

Presidencia del Consejo de Ministros. (2013). Política nacional de modernización de la gestión pública. Lima: Autor. 